\title{
Recurrent skin peeling following Kawasaki disease
}

\author{
Colin Michie, Veronica Kinsler, Robert Tulloh, Sue Davidson
}

\begin{abstract}
Long term follow up of 259 cases of Kawasaki disease led to the observation that $11 \%$ of children have episodes of recurrent peeling of the skin for several years after their recovery. These events were usually associated with an upper respiratory tract infection and were distinct from a recurrence of Kawasaki disease. Repeeling was significantly less frequent in children who had suffered coronary artery dilatation and was more frequently seen in those with nasal staphylococcal colonisation. The mechanism for this phenomenon is unclear, but it has been observed in a number of other conditions caused by infectious agents and their toxins. Paediatricians need to be aware of this phenomenon which is distinct from recurrence of Kawasaki disease.

(Arch Dis Child 2000;83:353-355)
\end{abstract}

Keywords: Kawasaki disease; skin peeling; keratolysis; long term follow up; Kawasaki disease sequelae

Children with Kawasaki disease (KD) usually have skin erythema which may involve the perineum or peripheries, one of the defining conditions of this condition. This is followed by peeling, keratolysis, or desquamation one to three weeks after the resolution of fever. A proportion of children may then develop recurrent episodes of skin peeling months or years after their initial illness. Similar desquamation has been reported in patients recovering from toxic shock syndrome and illness involving streptococcal toxins such as scarlet fever. ${ }^{1}$ We set out to document the phenomenon of repeeling in a cohort of children with $\mathrm{KD}$.

\section{Methods}

The frequency of repeeling was investigated in a cohort of 259 British patients who had KD after 1986; the study therefore covered 1239 patient years in total (table 1). Patients met five or six of the six criteria for the diagnosis of Kawasaki syndrome as defined by the American Centres for Disease Control. All patients had peeling as a component of their initial illness. The cohort was compiled from two sources. One hundred and seventeen successive patients and their parents attending a long term Kawasaki disease follow up clinic between 1996 and 1999 were asked prospectively as part of their routine clinic visits about the health of their skin and episodes of peeling. These patients had full documentation of their original illness available in the clinical notes. Nasal staphylococcal colonisation was checked in 35 of these patients during follow up clinic visits; all positive cultures were checked for toxin production using reverse passive latex agglutination kits (Unipath, Basingstoke, UK). Four patients had skin peeling observed during an admission to hospital for other reasons; clinical records of these admissions were reviewed carefully.

The remainder of the cohort was compiled using a retrospective questionnaire applied by a national patient help group for $\mathrm{KD}$ over the same time period. Of 195 questionnaires sent out, 142 fully completed answers were received. The original medical records were sought in 70 cases in which there were queries about aspects of the original diagnosis. The questionnaire covered the same areas as were reviewed in the prospective cohort. Data were analysed using a $\chi^{2}$ test.

\section{Results}

This patient group (table 1) was representative of the type of cohort reported by other authors
Paediatrics, Ealing

Hospital NHS Trust, Uxbridge Road,

London UB1 3HW, UK

C Michie

V Kinsler

Department of Paediatric Cardiology, Guy's and St Thomas's Hospital Trust, St

Thomas' St, London SE1 9RT, UK

$\mathrm{R}$ Tulloh

Kawasaki Support Group, 13 Norwood

Grove, Potters Green,

Coventry CV2 2FR, UK $S$ Davidson

Correspondence to:

Dr Michie

email: colinm@easynet.co.uk
Table 1 Comparison between the total cohort and those who suffered from repeeling

\begin{tabular}{lll}
\hline & Total cohort & Repeeling group \\
\hline Patient numbers & 259 & 29 \\
Patient age at presentation with KD (median and range) & 24 months (2-204) & $23(2-67)$ \\
Follow up time after illness (median and range) & 54 months (6-145) & $54(6-145)$ \\
Males/females & $159 / 100$ & $23 / 6$ \\
Patients with coronary aneurysm/s & 57 & $2^{\star}$ \\
Patients with previous atopic dermatitis & 203 & 23 \\
Patients with hearing damage or loss after KD & 5 & 3 \\
Patients referred to psychiatrist/psychologist & 14 & 73 of $15 \dagger$ \\
Number positive for staphylococcal colonisation & 19 of 35 & 3 of 13 \\
Staphylococcal cultures positive for any toxin production & 6 of 19 & 2 of 8 \\
Positive ASOT titres & 2 of 17 & 23 \\
Patients treated with immunoglobulin within 7 days of onset of initial KD & 217 & 23 \\
Patients treated with aspirin & 216 & 0 \\
Number of recurrent episodes of Kawasaki syndrome & 2 & 102 \\
Patient years studied & 1239 & 188 \\
Estimated number of repeeling episodes & 0 & 0
\end{tabular}

${ }^{\star} \mathrm{p}<0.05 ; \mathrm{tp}<0.001$. 
in a previous British study. ${ }^{2}$ No significant differences were detected between data collected in the prospective outpatient group and the retrospective questionnaire; in particular the proportions of repeeling detected by these two methods were the same. None of the patients suffered from skin peeling prior to their episode of $\mathrm{KD}$. This phenomenon would therefore appear to be linked in some way to their illness. None of the 190 siblings of the total patient cohort suffered from recurrent peeling, an observation supporting this statement. Repeeling episodes were most numerous in the first two years after diagnosis (139 episodes in the 29 patients); none was documented seven years after diagnosis. In the cohort 29 children suffered from recurrent peeling of their skin and two suffered from a recurrence of the full spectrum of Kawasaki disease. Recurrent Kawasaki disease in both cases was accompanied by prolonged high fever, enlarged lymph nodes, and by erythema of the skin for some seven to 14 days prior to peeling (as described in a Japanese cohort $^{3}$ ).

Using the admission data available for 187 cases, there was no correlation between acute phase measures of $\mathrm{KD}$, including platelet count (in all cases), white count (in all cases), eosinophil count (in 185 cases), or $\operatorname{IgE}$ concentration (in 42 cases) and the subsequent development of repeeling. All four children with repeeling during a hospital admission were admitted for an upper respiratory tract infection. In none of these cases was streptococci or staphylococci cultured from throat swabs, but on all occasions staphylococci were isolated from the nose (in the one inpatient tested, toxin screening of this organism showed no toxin production). Desquamation was observed on the second day of fever in one case, and between the third and fifth day of fever in the other three cases. None of the admissions showed evidence of erythema, conjuctivitis, or widespread lymphadenopathy and all responded rapidly to conservative measures or oral antibiotic for their infection. None had an increased eosinophil count or increase in urinary histamine during the period when their skin peeled.

\section{Discussion}

The recurrence of skin peeling in patients who have previously suffered from $\mathrm{KD}$ appeared to be a relatively common entity seen in $11 \%$ of patients, documented approximately 188 times in 1259 patient years in this cohort. These data suggested that this condition is distinct from recurrence of the disease. Desquamation in the initial illness followed inflammatory changes in the skin of the hands and feet whereas repeeling developed within a few days of the beginning of a minor illness, or simultaneous with it, and was not associated with a recurrence of skin inflammation. Repeeling may persist for several years after the episode of Kawasaki disease before resolving with no long term sequelae. The areas of skin which repeeled were seldom the most severely affected areas during the first episode of $\mathrm{KD}$.
With few exceptions the patients with repeeling were children who had not developed measurable coronary artery dilatation and therefore were thought by clinicians to have a more mild illness. A tendency to repeel did not relate to the timing of the commencement of therapy with immunoglobulin, the use of aspirin, or the timing of hospitalisation. The majority of children with repeeling were otherwise healthy. However, it was interesting to observe that children with notable behavioural sequelae or hearing problems following Kawasaki disease were observed at a higher frequency in the cohort which had recurrent desquamation. ${ }^{4-6}$ Distinctive clinical patterns such as this presumably represents a heterogeneity in the disease process, the host response, or both.

The mechanism of desquamation in $\mathrm{KD}$ is not clear. Following the observation that there was a disproportionately high rate of staphylococcal carriage in children with recurrent desquamation one may propose that release of superantigens or toxins from resident or colonising bacteria may play some role. A similar process has been proposed in two cases of recurrent perineal erythema, in whom the recurrence of erythema in conjunction with pharyngitis was attributed to toxin producing streptococci colonising the pharynx. ${ }^{7}$ Challenging this rather convenient model is the observation that the majority of staphylococcal cultures in this cohort collected showed no toxin production. Alternatively recurrent peeling may represent abnormal apoptosis of epithelial cells, ${ }^{8}$ or abnormal cytokine release from resident immune cells in the skin. This latter process could include $\mathrm{T}$ cells rendered anergic by the initial illness (as has been proposed for other illnesses involving bacterial toxins and superantigens ${ }^{9}$ ). The similarity of this peeling rash to that seen in scarlet fever or other streptococcal infections and toxic shock syndrome, or the systemic administration of interferon alfa suggests a more generalised mechanism rather than a specific one.

In conclusion paediatricians and dermatologists should be aware of the phenomenon of repeeling following Kawasaki disease, and the importance of distinguishing between recurrent disease and this milder, and seemingly benign complication. Children with Kawasaki disease should have some element of long term follow up or clear information sheets in order to reassure parents about this non-cardiac complication of this disorder.

Ealing Paediatric Courses funding supported this study. We should like to thank Cathy Marriott and Mari Satou for their assistance in reviewing the Japanese literature, and for a review of the script by Professor Mike Dillon. We should also like to thank the families of patients who have helped with this study, and their clinicians for referring patients to the Kawasaki and their.

1 Leung DY, Meissner HC, Fulton DR, Murray DL, Kotzin BL. Schlievert PM. Toxic shock syndrome toxin-secreting Staphylococcus aureus in Kawasaki syndrome. Lancet 1993;342:1385-8.

2 Tizard EJ, Suzuki A, Levin M, Dillon M. Clinical aspects of 100 patients with Kawasaki disease. Arch Dis Child 1991;66:185-8. 
3 Nakamura Y, Oki I, Tanihara S, Ojima T, Yanagawa H. Cardiac sequelae in recurrent cases of Kawasaki disease: a comparison between the initial episode of the disease and recurrence in the same patients. Pediatrics 1998;102:E66.

4 King J, Schlieper A, Birdi N, Capelli M, Korneluk Y, Rowe P. Kawasaki syndrome and its effects on cognitive, academic and behavioural function. A case control study. Pediatr Res 1996;39:18A.

5 Sundel RP, Newburger JW, McGill T, et al. Sensorineural hearing loss associated with Kawasaki disease. I Pediat 1990;117:371-7.
6 Michie CA, Tulloh R, Mills G, Davidson S. Kawasaki syndrome may be associated with behavioural and learning syndrome may be associated with behav

7 Manders SM, Heymann WR, Atillasoy E, Kleeman J, Schlievert PM. Recurrent toxin-mediated perineal erythema. Arch Dermatol 1996;132:57-60.

8 Viard I, Wehrli P, Bullani R, et al. Inhibition of toxic epidermal necrolysis by blockade of CD95 with human intravenous immunoglobulin. Science 1998;281:490-3.

9 Michie CA, Cohen J. Clinical significance of superantigens. Trends Microbiol 1998;6:61-5.

\section{EUROPE CALLING}

\section{Coincidence is not causality - a principle which needs regular rediscovery}

After the long debates on the safety of pertussis whole cell vaccines had finally been settled (they had been accused of causing severe brain damage in a subset of vaccinated infants and young children), new trouble recently arose with reports on the perceived side effects of immunisation. ${ }^{2-4}$

It was not enough that for almost two decades hundreds of thousands of children had to suffer unnecessarily from whooping cough and its complications after scares among lay persons and professionals fuelled by case reports and reports in the media on serious post immunisation illnesses. This led to under or non-use of the vaccine in many European countries, primarily Germany, Italy, and Sweden. The simple principle that "coincidence is no proof of causality" has been again neglected and new fears have been raised by studies that have inappropriate conclusions-for example, colitis and pervasive developmental disorders (autism), diabetes mellitus with onset at early age, and demyelinating diseases have been attributed to measles, mumps, and rubella (MMR) immunisation in the $\mathrm{UK},{ }^{4}$ invasive Haemophilus influenzae type B (Hib) diseases in Finland, ${ }^{35}$ and hepatitis B in France. ${ }^{2}$

The consequences were far reaching and have led to the decline of MMR immunisation rates in the UK and suspension of routine school-based vaccination of adolescents against hepatitis B in France, even though scientific evidence was against the allegations. ${ }^{67}$ The Hib/diabetes issue, fortunately, has not had any recognisable effect on Hib vaccine coverage in Finalnd or any other European country.

The reasons for the general public's enormous and, it seems, ever increasing interest in vaccine damage are multifactorial and rather easy to understand: an effective programme of vaccination leads to a reduced rate of occurences of the disease it seeks to prevent. Thus, the public fear of a serious illness - for example, poliomyelitis, becomes theoretical in the lack of actual cases, and the acceptance of an immunisation programme decreases. In parallel, public interest increasingly focuses on the perceived (or real) side effects of the vaccine. This is the dilemma we have to fight against.
Ideally, all parties concerned with vaccine safety-that is, the patients or their parents, legal authorities, manufacturers, and immunising physicians, should have a common interest in discriminating between coincidence and causal link of post-immunisation adverse events in order to maintain confidence in our successful vaccination programmes. Unfortunately, this is hardly ever the case. On 23 May 2000, despite overwhelming evidence that there is no causal link between multiple sclerosis and other demyelinating diseases and hepatitis $\mathrm{B}$ immunisation, ${ }^{7}$ the French Ministry of Health announced that it had decided to give financial compensation to eight individuals who had claimed serious medical problems after hepatitis B immunisation. The Ministry of Health argued that there is a causal link between hepatitis B immunisation and serious medical problems-French journalists picked up on this and spread the news that "new data" indicated a causal link (CA Siegrist, personal communication). There is no such new data and I wonder what the next story will be. (By the way, I observed side effects in both my daughter and my son within two weeks of their first dose of MMR at the age of 12 months: they both said "Daddy" for the first time shortly afterwards and also soon started to walk.)

ULRICH HEININGER European Associate Editor

1 Cherry JD. Pertussis vaccine encephalopathy: it is time to recognize it as the myth that it is. $7 A M A 1990 ; 263: 1679-80$.

2 Herroelen L, de Keyser J, Ebinger G. Central nervous system demyelination after immunisation with recombinant hepatitis B vaccine. Lancet 1991;338: after im

3 Classen JB, Classen DC, White H. Association between type 1 diabetes and Hib vaccine. BM7 1999;319:1133.

4 Wakefield AJ, Murch SH, Anthony A, et al. Ileal-lymphoid-nodular hyperplasia, non-specific colitis, and pervasive developmental disorder in children. Lancet 1998;351:637-41

5 Karvonen M, Cepaitis Z, Tuomilehto J. Association between type 1 diabetes and Haemophilus influenzae type $\mathrm{b}$ vaccination: birth cohort study. $B M \mathcal{F}$ 1999;318:1169-72

6 Taylor B, Miller E, Farrington CP, et al. Autism and measles, mumps, and rubella vaccine: no epidemiological evidence for a causal association. Lancet 1999;353:2026-9.

7 Hall A, Kane M, Roure C, Meheus A. Multiple sclerosis and hepatitis B vaccine? Vaccine 1999;17:2473-5. 